\title{
Contemporary methods of radiosurgery treatment with the Novalis linear accelerator system
}

\author{
Joseph C. T. Chen, M.D., Ph.D., ${ }^{1}$ Javad Rahimian, Ph.D., ${ }^{2}$ Michael R. Girvigian, M.D., ${ }^{2}$ \\ AND Michael J. Miller, M.D. ${ }^{2}$
}

Departments of ${ }^{\prime}$ Neurological Surgery and ${ }^{2}$ Radiation Oncology, Southern California Permanente Medical Group, Los Angeles, California

\begin{abstract}
$\checkmark$ Radiosurgery has emerged as an indispensable component of the multidisciplinary approach to neoplastic, functional, and vascular diseases of the central nervous system. In recent years, a number of newly developed integrated systems have been introduced for radiosurgery and fractionated stereotactic radiotherapy treatments. These modern systems extend the flexibility of radiosurgical treatment in allowing the use of frameless image-guided radiation delivery as well as high-precision fractionated treatments. The Novalis linear accelerator system demonstrates adequate precision and reliability for cranial and extracranial radiosurgery, including functional treatments utilizing either framebased or frameless image-guided methods. (DOI: 10.3171/FOC-07/12/E4)
\end{abstract}

KEY WORDS • radiosurgery • linear accelerator • image guidance • stereotaxy

$\mathrm{R}$ ADIOSURGERY stands as a unique field in medical practice, presently encompassing the disciplines of neurosurgery, radiation oncology, and medical physics. Although radiosurgical treatment initially met with substantial skepticism from neurosurgeons as well as radiation oncologists, its utility has become so clear as to place it as a key component in the neurosurgical armamentarium. From its earliest beginnings, radiosurgery has been a field dependent on and driven by technology, and arguably most of the advancements in the associated technology, clinical methods, and outcome data have been contributed by members of the neurosurgical community. In the last decade, improvements in imaging, computing, and understanding of disease processes have led to the development of a number of turnkey systems for addressing a broad array of radiosurgery methods.

The Novalis system (BrainLAB AG) is a purpose-built LINAC system for the delivery of stereotactic radiosurgery. It is capable of both cranial and extracranial treatments using frame and frameless techniques. The Novalis device represents a contemporary evolution of the classic LINAC paradigm - a gantry-based device that uses multiple noncoplanar arcs or multiple intersecting static fields to deliv-

Abbreviations used in this paper: $\mathrm{CT}=$ computed tomography; IMRT = intensity-modulated radiation therapy; IMRS = intensitymodulated radiosurgery; LINAC $=$ linear accelerator; $\mathrm{MR}=$ magnetic resonance. er conformal treatment. This device is characterized by great flexibility in dose planning and delivery.

In addition to the classic radiosurgery paradigm of frame-based, forward-planned, single-fraction cranial treatment, additional modes of therapy and planning are possible and can be used in various combinations to suit the needs of a variety of disease indications and workflow issues. The scope of indications can be broadened to lesions not typically addressable by single-fraction framebased techniques. This flexibility is also accompanied by greater complexity in the clinical decision-making and treatment processes. In this paper the authors describe in brief the operational methods used at our center.

\section{Device Description}

The Novalis LINAC system is specifically manufactured for high-precision radiosurgery (Fig. 1). The basis of this device is a lightweight gantry-based single-energy (6 MV) LINAC, which allows the device to have a lower mass than typical general-purpose LINACs and thus facilitates gantry isocentricity. The specification of the gantry isocentricity for this device is a linear 3D error of $\geq 0.3 \mathrm{~mm}$. In addition to the LINAC itself, the system includes components consisting of treatment planning software, patient positioning hardware, and collimation hardware.

For high-precision radiation treatments, errors in energy output and spatial positioning must be minimized. Output factor measurements for Novalis with both cones and mul- 


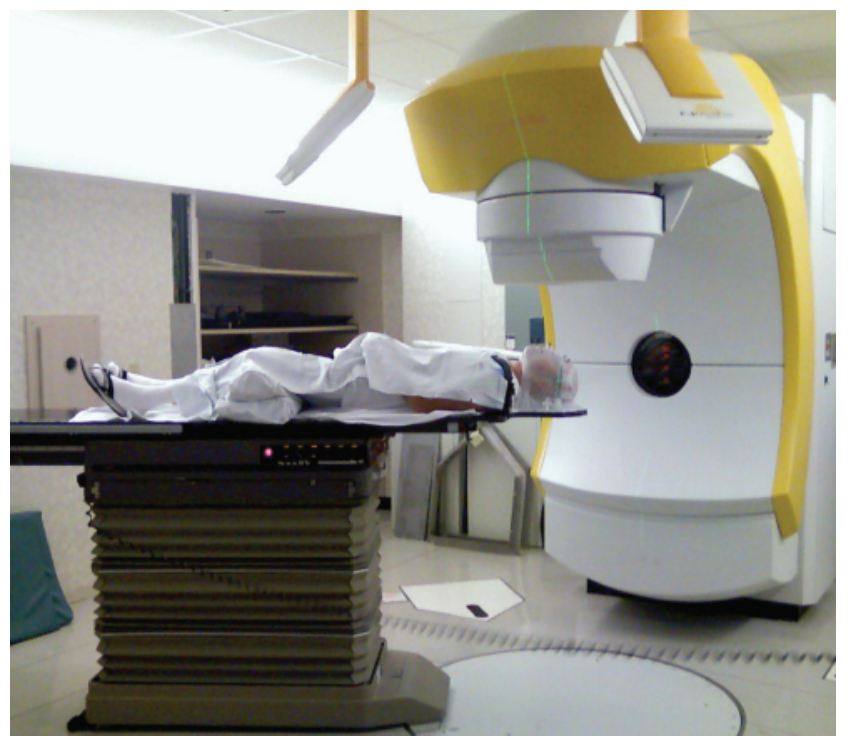

FIG. 1. Photograph featuring the Novalis LINAC suite at the Southern California Kaiser Regional Radiation Center.

tileaf collimation have been described by Yin et al. ${ }^{11} \mathrm{We}$ have performed detailed measurements of output factors as part of our routine quality assurance process and found our results to be in close agreement with data in published reports. Dose output from the device is regularly checked using diode detectors. In addition, our center participates in the thermoluminescent dosimeter remote monitoring program sponsored by the American Association of Physicists in Medicine. Since commissioning this device in 2002, all measurements of dose output have been within $1 \%$ of expected values.

We have also independently investigated the geometric accuracy of this device in detail, assessing the entire chain of image and target acquisition as well as radiation delivery for radiosurgery. We have found that the overall 3D root mean square geometric accuracy is $0.6 \pm 0.37 \mathrm{~mm} .{ }^{8}$ Similar results have been reported by others. ${ }^{9}$ We have found that the greatest source of uncertainty in treatment lies in the errors associated with imaging and fiducialization rather than with factors related to radiation delivery and patient positioning.

Several options are available for patient positioning and immobilization. Frame-based immobilization can be accomplished by using the proprietary BrainLAB stereotactic frame or the Radionics X-Knife frame, as well as the Leksell G-type stereotactic frame (Elekta). BrainLAB supplies adapters that allow mating each of these frames with the LINAC couch mount as well as specific target positioning overlay boxes. Frameless treatments utilizing image-guidance can be accomplished with a wide array of immobilization devices supplied by BrainLAB or other manufacturers. The image-guided module consists of a stereoscopic kilovolt $\mathrm{x}$-ray system using amorphous silicon detectors coupled with a robotically actuated treatment table capable of correcting each of 6 degrees of freedom. These hardware components are then linked together using a number of software tools that enable dose planning, patient positioning, and dose delivery.

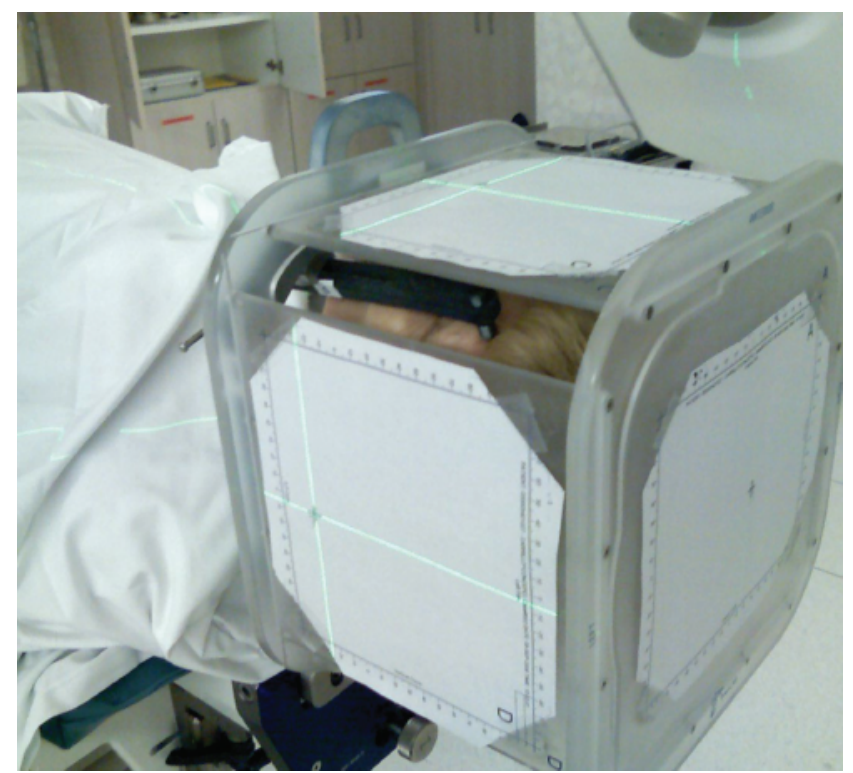

FIG. 2. Photograph depicting the target positioning box with computer-generated overlays for room laser alignment.

\section{Treatment Process}

\section{Frame-Based Radiosurgery}

Stereotactic frame-based immobilization and localization have long been the hallmark of the radiosurgery method. At our center, we apply the stereotactic frame after administering a complete local anesthetic. Typically, patients are brought into the procedure suite in a wheel chair or on a gurney. The appropriate scalp and forehead regions are then prepped with alcohol solution. Lidocaine $(1 \%)$ is then infiltrated into the scalp at the planned pin sites. Once appropriate anesthesia is achieved, the stereotactic frame is then applied to the head and held in place using 4-point pin fixation. The pins are tightened with a torque wrench to 25 $\mathrm{cN} \times \mathrm{m}$, which is adequate to affix the frame securely to the head without distorting the frame.

A high-resolution fiducial CT scan (GE Lightspeed, GE Medical Systems) is then obtained with axial 0.6- or 1.25mm-thick slices by using a BrainLAB or Radionics N-bar localizer. The CT scan covers the distance from the vertex to the base of the skull in all cases, as CT data are used for the calculation of depth dosage. In select arteriovenous malformation cases, stereotactic angiography is used for localization with the Radionics SGV or BrainLAB localizer as well as CT and axial MR imaging.

High-resolution fine-cut MR images are obtained before the day of treatment. At our facility, a GE Signa Excite 1.5$\mathrm{T}$ unit (GE Medical Systems) is used for MR imaging. Axial images of 1-mm-thick 3D spoiled gradient-echo pulse sequences with or without Gd enhancement are typically used for tumor and functional treatments. Sequences with fat suppression are especially useful for skull base plans when adipose tissue can obscure enhancing tumor. Fine-cut images allow a near isotropic 3D reconstruction of the image data. Additional MR imaging sequences such as 3D fast imaging employing steady-state acquisition as well as data from other axial imaging modalities such as pos- 


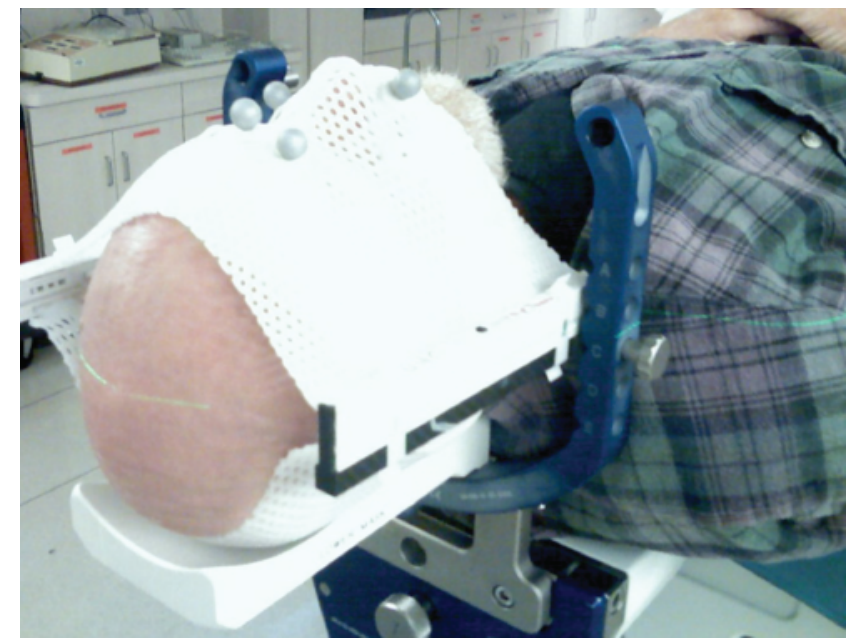

FIG. 3. Photograph showing the BrainLAB U-PLAST mask fixation system.

itron emission tomography can be simultaneously imported and used for treatment planning.

The BrainLAB system requires all treatment planning to be based on a CT data set as the primary reference point to which MR imaging and other data are fused. This strategy mitigates uncertainties due to magnetic field inhomogeneities and other artifacts in MR imaging, which can vary depending on scanning protocols and from patient to patient. ${ }^{2}$ Data from MR imaging and CT studies are then fused using an automatic fusion algorithm that relies on general statistical dependence between voxel intensities. ${ }^{6}$ Treatment planning starts with the identification of target volumes and at-risk structures. These are contoured to allow the calculation of dose-volume histograms. The radiosurgery team, using one of the several treatment modes supported by the device, then develops a treatment plan. The treatment plans are reviewed and approved by a group consisting of a radiation oncologist, neurosurgeon, and medical physicist. These plans typically consist of between 3 and 7 noncoplanar arcs or between 5 and 10 convergent shaped beams.

After generating the treatment plan and appropriate quality control checks, radiation delivery is undertaken. Data from the planning station is then transferred to the LINAC via an integrated record-and-verify solution (IMPAC Medical Systems).

At our center, all single-fraction treatments are performed under the direct and immediate supervision of the neurosurgical and radiation oncology medical staff as well as a medical physicist and radiation therapist. The patient is brought into the treatment suite and positioned on the LINAC couch. The patient's head is then immobilized by attaching the stereotactic frame to the couch mount. A target-positioning box utilizing computer-generated templates is used to align the target into the isocenter via calibrated room lasers (Fig. 2). Microadjustments via the couch mount are then made to ensure precise alignment of the isocenter for each table angle.

After delivering the radiation dose, the stereotactic frame is removed from the patient's head. Dry dressings are

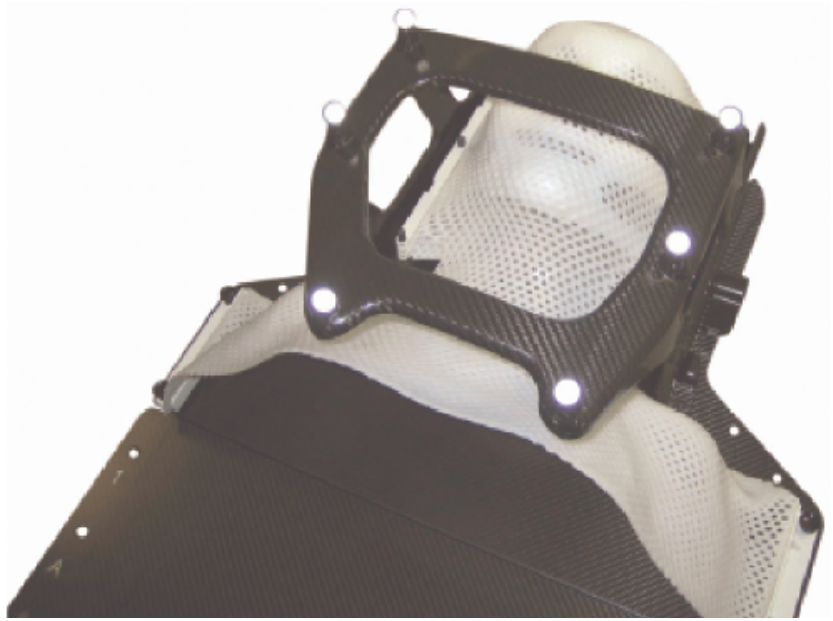

FIG. 4. Photograph illustrating the BrainLAB mask immobilization device with optical marking spheres for image-guided radiation therapy and image-guided radiosurgery.

placed, and the patient is discharged home. Typical treatment times range from $\sim 1$ hour for trigeminal neuralgia indications to $<20$ minutes per lesion for most other lesions.

Fractionated stereotactic radiotherapy, which typically ranges from 3 to 30 fractions, has been applied in the treatment of selected lesions. Such lesions are typically too large for single-fraction radiosurgery or are in contact with a special sensory nerve, such as the optic nerve, and have limited single-fraction dose tolerance. To accomplish these treatments, a special relocatable mask and/or headframe is used. At our center we use the BrainLAB U-PLAST mask system (Fig. 3), which consists of a rigid thermoplastic mesh that is molded to both the anterior and posterior surfaces of the head. A small bite bar is incorporated into the mask to reduce translation along the longitudinal axis. We use a Radionics GTC depth helmet to verify the patient position along the longitudinal axis of the mask. After fitting the mask, it is allowed to cure for at least 48 hours to allow for stabilization of the thermoplastic. A fiducial CT scan is then obtained while the patient is positioned in the mask. Planning and treatment delivery are then accomplished in the same fashion as for radiosurgery treatments. Due to uncertainty regarding patient repositioning, we have typically used this technique for full fractionation treatments and have typically assigned 3-mm margins for the treatment volume. Homogeneous dose plans covering the volume of tumor to the 90-95\% isodose line are typically used to further mitigate positioning uncertainty as well as to protect at-risk structures. Because of these issues, we now use image-guided radiosurgery methods for all fractionated stereotactic radiotherapy treatments.

\section{Image-Guided Radiosurgery}

The desire to treat lesions outside of the head and to use the highest precision dose delivery in the setting of fractionated stereotactic radiotherapy have led to the development of image-guided radiosurgery/radiotherapy. The introduction of image-based positioning systems integrated 


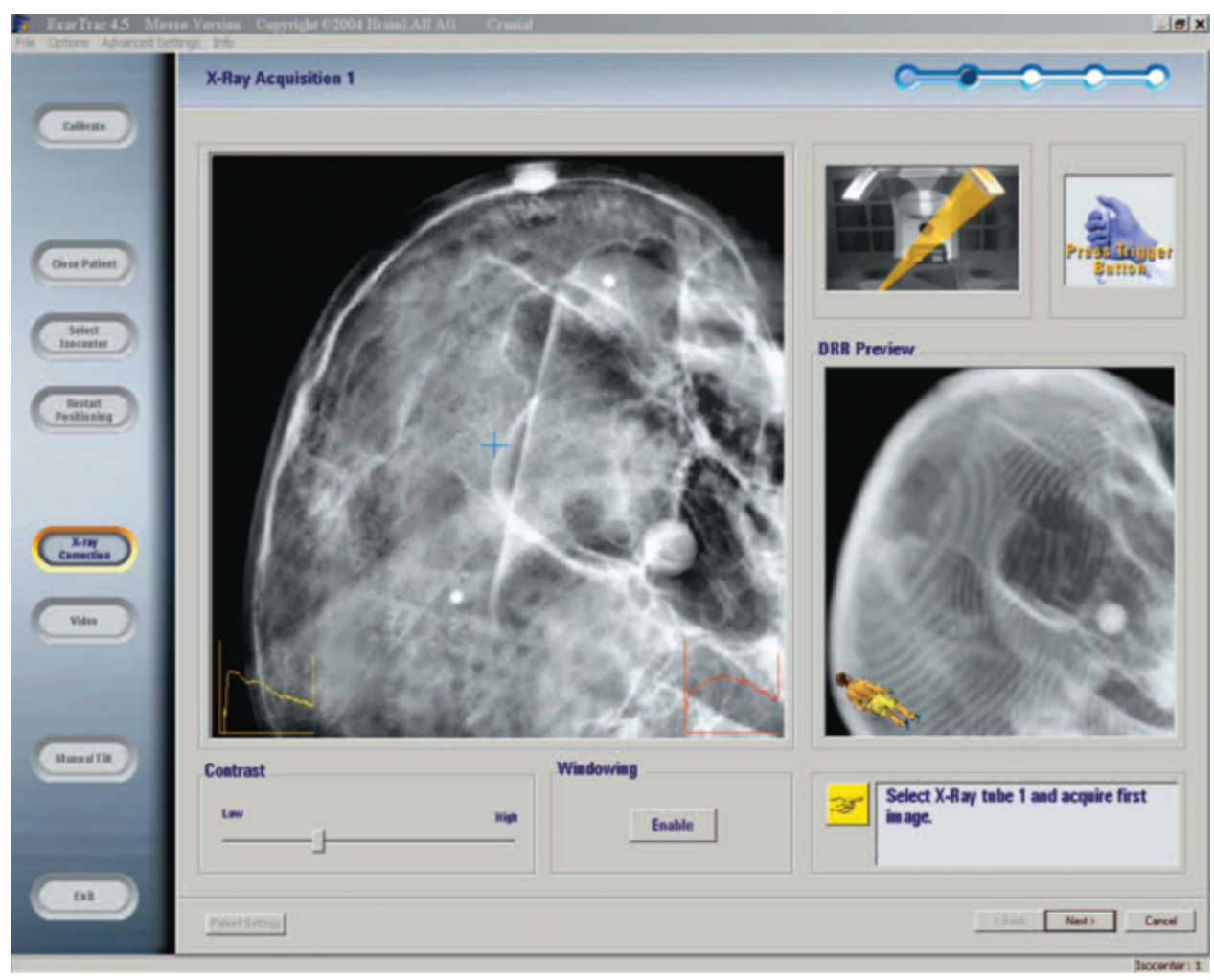

FIG. 5. Display screen from the ExacTrac patient positioning system demonstrating the kilovolt x-ray image (large image on left) and digitally reconstructed radiograph (small image on right) used for image fusion and the calculation of positioning offset.

with the treatment delivery device has added broad new capabilities to the radiosurgery community. In addition to extending the radiosurgery indications, frameless methods improve overall workflow by allowing imaging and treatment planning and delivery to be done at separate time frames and in different locations. Given that the accuracy of dose delivery for each fraction of the fractionated therapy is similar to that used for frame-based treatments, no additional treatment margin is required, and higher peak doses can be achieved within the tumor volume. These features can lead to superior tumor control and a reduction in the dose to adjacent critical structures.

The key concept is the ability to determine through imaging the exact position of the target relative to the device and then automatically make adjustments to compensate for initial positioning errors. Two general methods are used to accomplish this task: stereoscopic $\mathrm{x}$-ray imaging, such as that used in the Novalis LINAC system and CyberKnife (Accuray), and on-board cone-beam CT, such as that used in the Trilogy system (Varian Medical Systems), the Synergy and Axxess systems (Elekta), and the Oncor system (Siemens AG). The advantage of stereoscopic x-ray methods is the reduced radiation exposure for imaging as well as rapid image acquisition. On-board CT scanning has the advantage of better delineation of soft-tissue targets located remotely from osseous fiduciary points, which may mean a particular advantage for certain lesions located in the thoracic and abdominal cavities. For applications involving the spine and brain, however, it is our opinion that stereoscopic x-ray-based localization presently has signifi- cant advantages over on-board CT scanning from the standpoint of workflow and accuracy.

In addition to the stereoscopic x-rays, Novalis uses a real-time optical tracking system, which allows real-time tracking of externally marked points to accurately determine departures from correct patient positioning between x-ray imaging cycles.

Image-guided cranial procedures are performed using a mask immobilization system (Fig. 4). At the time of CT acquisition, rather than using an N-bar localizer, CT-visible optical marking spheres are used. Data are then digitally transferred to the planning station on which the optical markers are acquired. The planning process is then identical to conventional frame-based treatments.

After the planning process, multileaf collimator and dose control data are then exported via the treat and verify system to the LINAC control computer. A separate set of data incorporating digitally reconstructed radiographs based on the CT data is are exported to the Novalis ExacTrac patient positioning system.

Initial patient positioning is accomplished using the optical tracking markers to obtain a rough initial estimation of patient positioning. Generally, the optical markers alone are sufficient to place the target to within $3 \mathrm{~mm}$ of the correct position for cranial applications. The stereoscopic x-ray system is then used to determine the precise location of the target structure in space relative to the machine isocenter by comparing the acquired $\mathrm{x}$-ray images to the digitally reconstructed radiographs with an image-fusion algorithm (Fig. $5)$. Both linear and angular offsets are then calculated in 


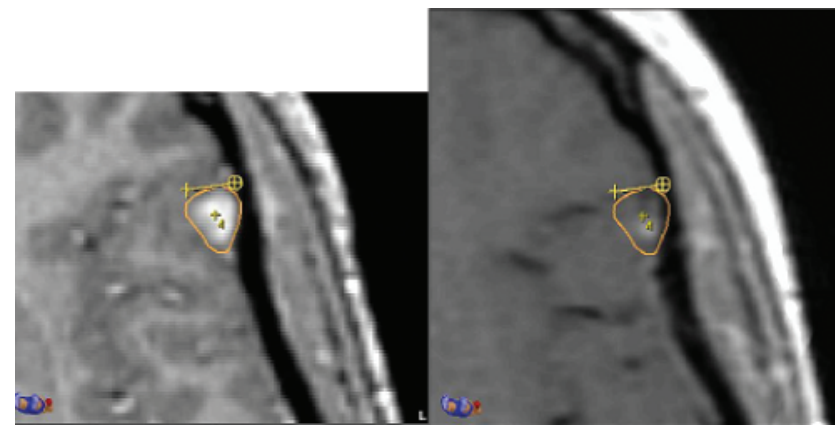

FIG. 6. Pretreatment (left) and 2-month posttreatment (right) images of a metastatic lung lesion treated with image-guided radiosurgery. A dose of 25 Gy was delivered to the isocenter with marginal coverage at $80 \%$. The measurement bar is $6.7 \mathrm{~mm}$ in length.

each of 6 dimensions of movement. The optical tracking system is reset according to the data from the kilovolt imaging system. The optical tracker is then used in conjunction with a robotic treatment table to precisely correct patient positioning. The process of kilovolt imaging with table shift correction is repeated until linear offsets are $<$ $0.5 \mathrm{~mm}$ and angular offsets are $<1^{\circ}$. Typically, only one shift iteration is needed to correctly position the target. Using phantom-based testing, we have found that overall the average linear positioning error is $\pm 0.6 \mathrm{~mm}$ in the context of $1.25-\mathrm{mm}$-thick CT slices. This accuracy is comparable to that achieved with a stereotactic frame and allows confident treatment of even small lesions (Fig. 6).

By using a rigid mask immobilization system, such as the U-PLAST mask, intrafraction motion is typically $<0.5$ $\mathrm{mm}$ in cranial cases. As such, reimaging is only typically performed after every 2 nd or 3rd arc or shaped beam. We have observed that intrafraction motion is highly dependent on the rigidity of the mask immobilizer, and with use of flexible masks, such as Aquaplast (WFR), intrafraction motion can be as high as $\pm 2 \mathrm{~mm}$. Such high intrafraction motion can lead to reduced confidence in the treatment and extended treatment times because of the repeated imaging and shift corrections necessary to maintain target positioning. Patient motion tends to be affected as well by habitus, with poorer motion control in obese patients in whom a thick layer of subcutaneous adipose tissue allows increased motion of osseous structures such as the skull or spine relative to the skin. Moreover, patients who suffer from significant pain, tremor, or labored breathing or who otherwise are unable to cooperate with the procedure should be considered for frame-based treatments or should be anesthetized for image-guided radiosurgery.

\section{Quality Assurance}

Given the complexity of LINAC devices for radiosurgery, a regular process of quality assurance is required, encompassing all aspects of the imaging and radiation delivery chain. Our quality assurance process includes regular MR imaging and CT checks. Gantry isocentricity and laser convergence are inspected on a daily basis by using the method of Winston and Lutz. ${ }^{10}$ Calibration of the image-guidance system is performed on a daily basis as well. Two steps are required: registration of the x-ray system with the optical tracking system and registration of the optical tracking system with the machine isocenter. The manufacturer provides two sets of calibration targets. The first target utilizes embedded radiopaque and optical markers to allow registration of the optical tracking subsystem with the kilovolt imaging subsystem (Fig. 6). Following this step, the optical system is in turn registered to the machine isocenter by visually placing a second calibrated device, which consists of an aluminum ingot etched with grooves that are manually aligned to the room lasers, to place the center of the target coincident with the gantry isocenter (Figs. 7 and 8). Optical tracking spheres embedded in this device in turn allow registration of the optical tracking system to the LINAC. This device can then be displaced arbitrarily on the treatment table to test the robotic table's ability to return the device to the machine isocenter.
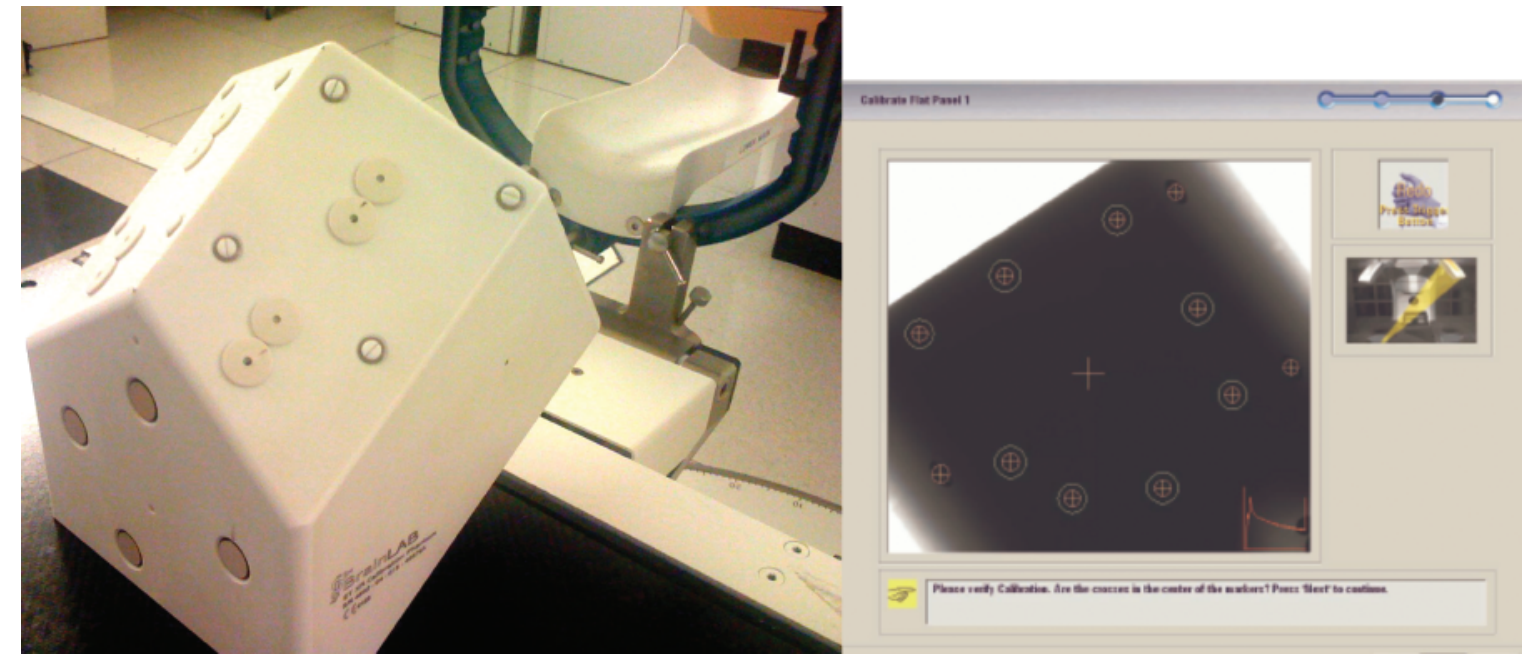

FIG. 7. A: Photograph depicting the calibration target for a kilovolt x-ray imaging system. B: ExacTrac screen after kilovolt imaging of the X-ray calibration target. Embedded calibration markers are automatically acquired by the system. 


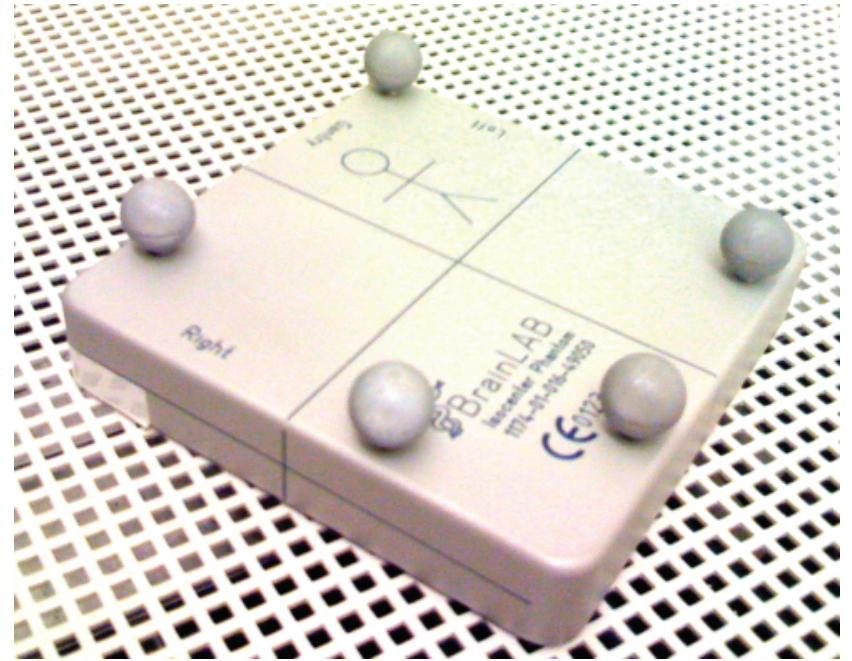

FIG. 8. Photograph showing the calibration target for the optical tracking system.

Return of the calibration device to the isocenter can be reliably accomplished to an error of not more than $0.1 \mathrm{~mm}$.

\section{Planning Methods}

A hallmark of modern LINAC-based radiosurgery systems is the great flexibility in treatment modes. The ability to choose from a number of modes allows the medical team the potential to treat the greatest diversity of pathological entities. Regardless of the precise therapeutic method, the goals are similar-delivering highly conformal energy to a radiographically defined target to achieve a consistent biological effect on the lesion while at the same time limiting the risk of potential complications to nearby structures.

\section{Forward Planning Methods}

In radiation physics, "forward planning methods" refer to techniques whereby the user actively designs the treat-

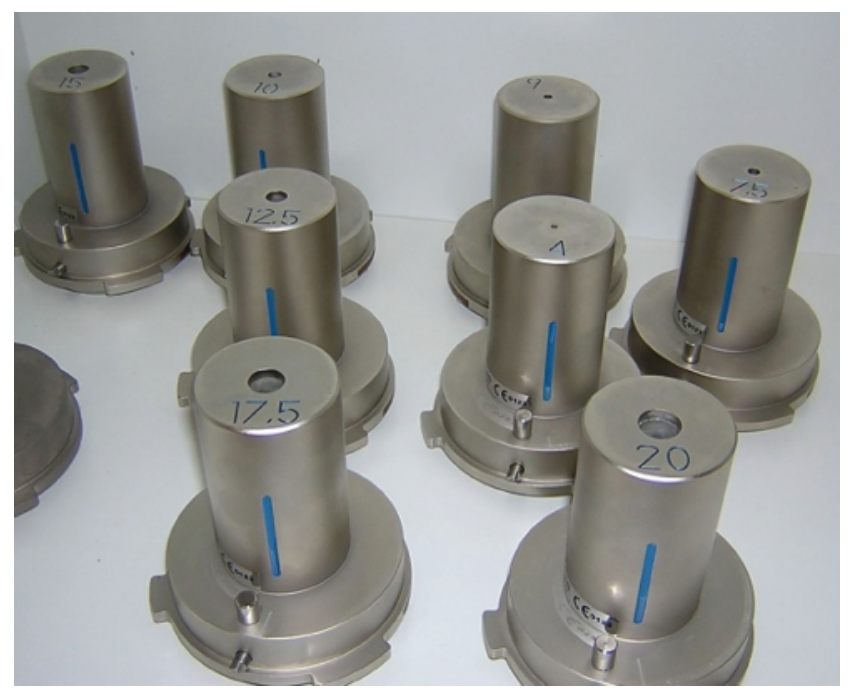

FIG. 9. Photograph demonstrating the standard complement of circular collimation cones.

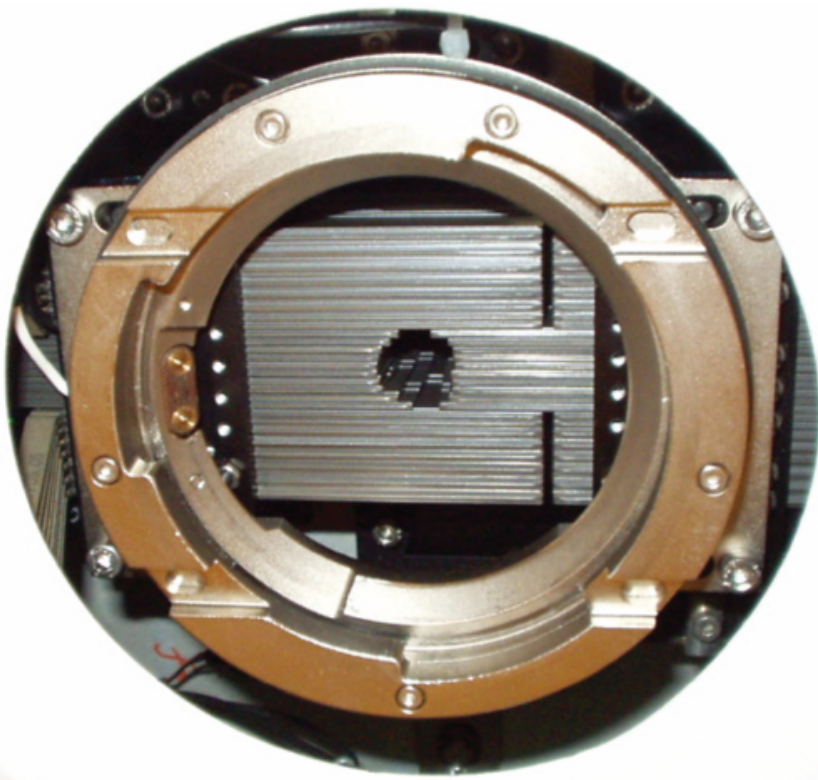

FIG. 10. Image of a Novalis micromultileaf collimator.

ment plan. With forward planning, computer assistance is used to calculate the resulting dose distribution from beam, arc, and isocenter placements determined by the user. An iterative process incorporating user-determined modifications to the treatment plans and recalculation of dose characteristics is performed to optimize dose, conformity, and exposure to at-risk structures. This system supports forward planning with circular collimation cones, static

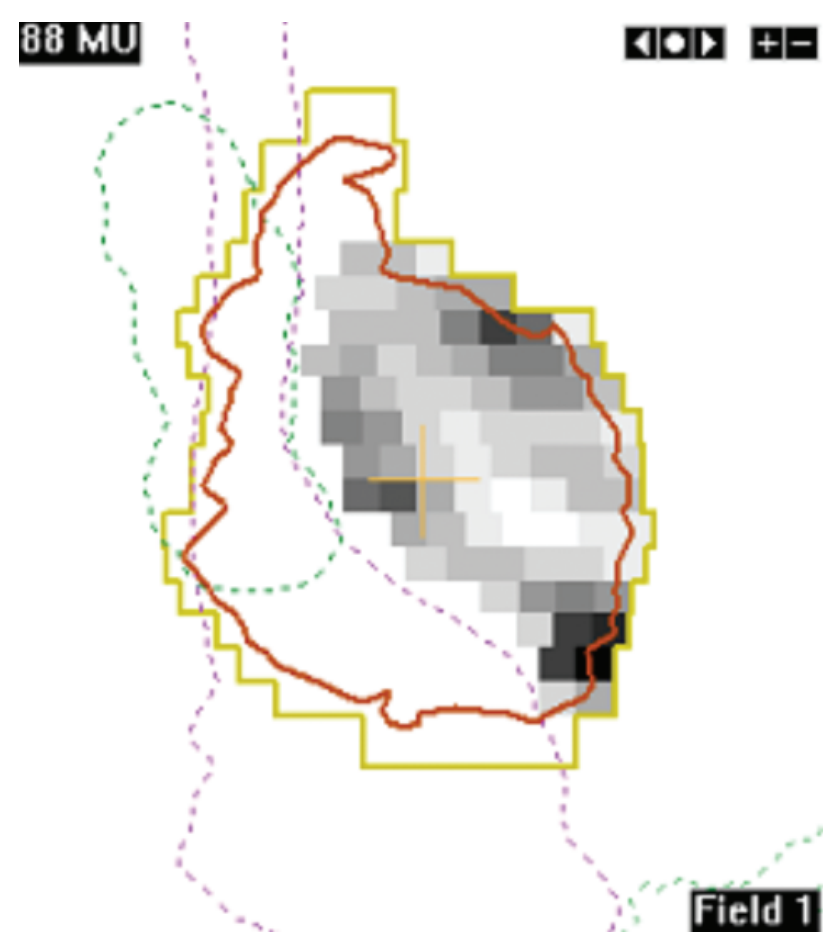

FIG. 11. Fluence map demonstrating the profile of a single beam of an intensity-modulated treatment plan. 

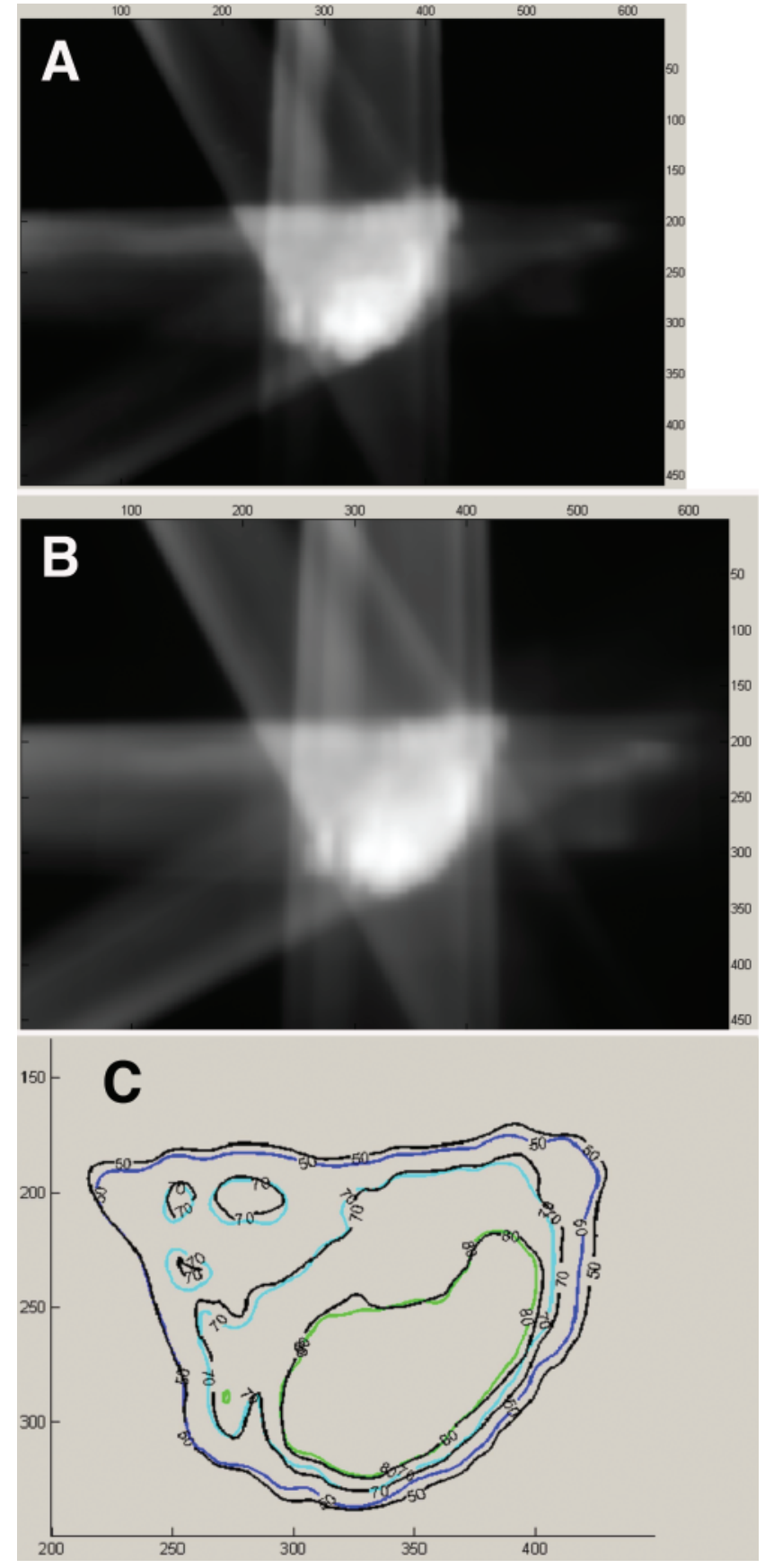

FIG. 12. A: Image depicting film quality assurance from an IMRT plan. B: Image showing the intended planned IMRT dose. $\mathrm{C}$ : Image demonstrating the fusion of the filmed (black) and planned (color) doses. Overall dose is within 3\% of planned for any arbitrary point.

shaped beams, and dynamically collimated arcs. As is typical with most LINAC-based planning systems, the prescription isodose line is typically at $80 \%$.

\section{Cone-Based Stereotactic Radiosurgery}

The Novalis device has available circular collimator cones. Cone collimators are typically supplied in 20-,
17.5-, 15-, 12.5-, 10-, 7.5-, 6-, and 4-mm widths (Fig. 9). Using these cones, one can generate conformal dose plans by combining noncoplanar arcs or static beams with differential weighting. We typically use cone-based methods for functional treatments and small-volume metastatic tumors. Larger lesions with irregular topologies are treated using multileaf collimator methods, simplifying the planning and delivery process.

\section{Static Shaped Beams}

This method utilizes the multileaf collimator to shape the treatment beam to match the profile of the target (Fig. 10). Multiple convergent shaped beams are used to achieve conformity. Static shaped beams are most appropriate in stereotactic radiosurgery for irregular lesions located close to sensitive structures at risk. We have also used this method primarily for extracranial body treatments. Finetuning of a dose is achieved by manual positioning of the collimator leaves and differential beam weighting. The Novalis multileaf collimator consists of 26 leaf pairs, with the centralmost 14 leaves having a 3-mm width, the intermediate 6 leaves having a $4.5-\mathrm{mm}$ width, and the peripheral 6 leaves having a 5.5-mm width. The maximal field size is $10 \times 10 \mathrm{~cm}$.

\section{Dynamic Conformal Arcs}

The method of dynamic conformal arcs combines the use of arc-based radiosurgery with dynamic collimation such that the beam shape changes during gantry rotation to follow the profile of the target structure. The Novalis device allows for collimator shape changes for every $10^{\circ}$ of gantry rotation. This method generally creates plans with high conformity and homogeneity. Typically between 3 and 5 arcs are used for each lesion isocenter, depending on the tumor volume, topology, and pathological features. At our facility, we have found that this treatment mode is suitable for the majority of neoplastic and vascular lesions that are treated. An advantage of this method of treatment is its speed: rapid planning and treatment are possible. Even for large lesions with complex shapes, treatments can be delivered in $<25$ minutes.

\section{Inverse Planning: IMRT and IMRS}

Intensity-modulated radiation therapy or IMRS implies a number of differences in operation compared with the previously discussed treatment modalities. It is a method whereby "inverse planning methods" incorporating powerful computer algorithms are used to design an optimized treatment plan that is constrained by parameters defined by the user. Inverse planning differs from so-called forward planning methods in that the computer passively calculates the dose in response to active planning from the operator. The method of IMRT or IMRS is analogous to the method of CT imaging. In CT imaging, a homogeneous x-ray beam is used to deliver energy to the structure to be imaged. The exit dose is nonuniform and dependent on variations of density within the imaged structure. In contrast, IMRT/IMRS delivers a nonuniform energy beam to the target in such a manner as to obtain a relatively uniform dose pattern within the target volume. In practice, dose constraints are made to maximize the dose to the target while minimizing the 


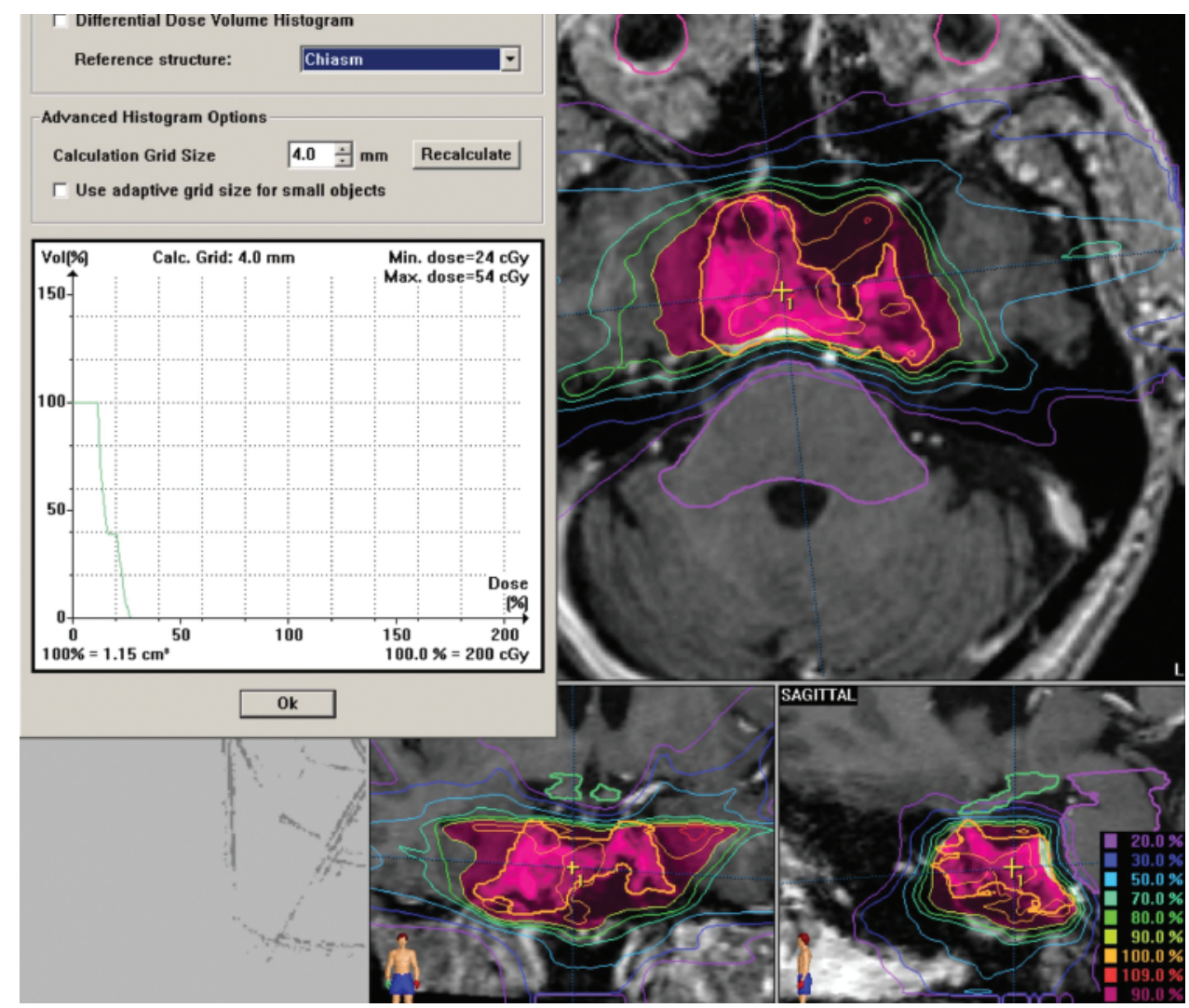

FIG. 13. Image depicting a dose plan for a clivus chordoma treated with IMRT, including excellent protection of the chiasm and brainstem.

dose to adjacent organs at risk. The final IMRT/IMRS plan best accommodates the tradeoffs between target coverage and the dose to at-risk organs as determined by the user.

BrainLAB IMRT/IMRS plans start with a basic static conformal beam plan with manually placed beam positions. The number of beams used depends on the size and location of the target in relation to adjacent at-risk structures. At our center, we typically use between 5 and 10 beams. Thereafter, the IMRT/IMRS planning mode is entered, and dose constraints are placed on the target and at-risk structures. A computer-generated treatment plan is then produced through an optimization routine that adjusts the intensities across the beam profile. Following beam placements, the IMRT/IMRS planning mode is entered and dose constraints are placed on the target and at-risk structures (Fig. 11). In this manner, greater treatment conformity can be achieved in comparison to other treatment modes.

Intensity-modulated radiation therapy or IMRS utilizes complex computational models whereby target volumes and at-risk structures are assigned quantitative values corresponding to maximal and minimal acceptable doses. For example, for a clivus chordoma, one may want to assign a total tumor margin dose of not less than $70 \mathrm{~Gy}$ over 30 fractions while limiting brainstem exposure to no more than 54 Gy. Inverse planning algorithms then determine the optimal intensity profile for each beam. Several algorithms are commonly used by different vendors. BrainLAB uses a dynamically penalized likelihood algorithm that has the advantage of lower computational demands with high ac- curacy. ${ }^{7}$ This feature allows the user to run multiple IMRT plans within the space of several minutes to adjust dose constraints to the target and at-risk organs.

Intensity-modulated radiation therapy or IMRS requires extensive quality control measures to ensure accuracy of dose delivery. ${ }^{1,5}$ This quality assurance method specifically addresses potential errors in dose calculation as well as in treatment delivery such as those in gantry/table isocentricity and collimator leaf movements. At our center we have used both film-based quality assurance as well as diode array methods. The LINAC is run to deliver the planned dose to x-ray film or a diode array detector (MapCHECK, Sun Nuclear). The delivered dose is then evaluated and compared with the intended dose (Fig. 12). A dose delivery error of $<3 \%$ is considered to be an adequate margin of error.

Because of the extensive quality assurance measures demanded by this technique as well as the additional time needed for dose delivery, we have reserved its use for cases in which satisfactory plans are unlikely to be realized using forward planning methods.

Intensity-modulated radiation therapy is particularly useful in cases in which there is a large concave surface on a lesion that envelops an at-risk structure or a high dose escalation is needed for radioresistant tumors located close to critical structures, for example, a chordoma (Fig. 13). In addition, IMRT/IMRS may be useful for salvage treatments in which high radiation doses have been previously delivered to the region of the treatment volume. 


\section{Determination of Treatment Methods}

In practice, the variety of treatment modalities can be very helpful in planning difficult cases, particularly those in which single-fraction radiosurgery is required near at-risk structures. Extensive experience with all of the treatment modes, however, is needed to generate plans using forward planning methods in an expeditious fashion. By routinely producing multiple plans using different treatment modes, we have gleaned important insights regarding the strengths and weaknesses of each approach.

The new generation of radiosurgery devices has dramatically extended the indications for high-conformity radiation methods; however, such flexibility comes with greater demands on quality control and clinical judgment because of the greater technical demands of the treatment and the increasing complexity of diseases. We have also found that it is critical that medical physicists and radiation oncologists as well as neurosurgeons take an active part in creating plans and supervising treatments to avoid potential complications and errors. An integrated group of specialized medical and technical staff is critical for a process of continuous improvement and quality control.

\section{Conclusions}

Modern LINAC technology has made great strides since the initial reports of Betti and Colombo and their colleagues. ${ }^{3,4}$ Advancements in computing technology have allowed automation of many aspects of LINAC radiosurgery planning and treatment. Therapies can be delivered in an entirely noninvasive fashion with high precision even for the smallest targets. A comprehensive system of quality control measures is critical to ensure both dose and spatial accuracy when using the newer generation devices. The additional flexibility in treatment modalities allows the extension of radiosurgery indications beyond what is possible with single-fraction frame-based methods. With this additional capability, radiosurgery treatment groups should consist of a core of specialized and experienced team members to deal with the fine points of complex pathological entities and treatment.

\section{Disclaimer}

None of the authors has a financial or consulting relationship with BrainLAB, AG or any other manufacturer listed in this paper.

\section{References}

1. Agazaryan N, Solberg TD, DeMarco JJ: Patient specific quality assurance for the delivery of intensity modulated radiotherapy. J Appl Clin Med Phys 4:40-50, 2003

2. Bernstein MA, Huston J III, Ward HA: Imaging artifacts at 3.0T. J Magn Reson Imaging 24:735-746, 2006

3. Betti O, Derechinsky V: [Multiple-beam stereotaxic irradiation.] Neurochirurgie 29:295-298, 1983 (Fr)

4. Colombo F, Benedetti A, Pozza F, Zanardo A, Avanzo RC, Chierego G, et al: Stereotactic radiosurgery utilizing a linear accelerator. Appl Neurophysiol 48:133-145, 1985

5. Galvin JM, Ezzell G, Eisbrauch A, Yu C, Butler B, Xiao Y, et al: Implementing IMRT in clinical practice: a joint document of the American Society for Therapeutic Radiology and Oncology and the American Association of Physicists in Medicine. Int J Radiat Oncol Biol Phys 58:1616-1634, 2004

6. Grosu AL, Lachner R, Wiedenmann N, Stärk S, Thamm R, Kneschaurek P, et al: Validation of a method for automatic image fusion (BrainLAB System) of CT data and 11C-methionine-PET data for stereotactic radiotherapy using a LINAC: first clinical experience. Int J Radiat Oncol Biol Phys 56:1450-1463, 2003

7. Llacer J, Solberg TD, Promberger C: Comparative behavior of the dynamically penalized likelihood algorithm in inverse radiation therapy planning. Phys Med Biol 46:2637-2663, 2001

8. Rahimian J, Chen JC, Rao AA, Girvigian MR, Miller MJ, Greathouse HE: Geometrical accuracy of the Novalis stereotactic radiosurgery system for trigeminal neuralgia. J Neurosurg 101 (3 Suppl):351-355, 2004

9. Solberg TD, Goetsch SJ, Selch MT, Melega W, Lacan G, DeSalles AA: Functional stereotactic radiosurgery involving a dedicated linear accelerator and gamma unit: a comparison study. J Neurosurg 101 (3 Suppl):373-380, 2004

10. Winston KR, Lutz W: Linear accelerator as a neurosurgical tool for stereotactic radiosurgery. Neurosurgery 22:454-464, 1988

11. Yin FF, Zhu J, Yan H, Gaun H, Hammond R, Ryu S, et al: Dosimetric characteristics of Novalis shaped beam surgery unit. Med Phys 29:1729-1738, 2002

\footnotetext{
Manuscript submitted September 12, 2007.

Accepted October 29, 2007.

Address correspondence to: Joseph C. T. Chen, M.D., Ph.D., Southern California Permanente Medical Group, Department of Neurological Surgery, 1505 North Edgemont Street, Fourth Floor, Los Angeles, California 90027. email: jctchen@yahoo.com.
} 
Author Names, et al. 